WUE-ITP-98-012

UWThPh-1998-14

HEPHY-PUB 691/98

hep-ph/9804306

\title{
Spin Correlations in Production and Decay of Charginos
}

\author{
G. Moortgat-Pick ${ }^{a}, \mathrm{H} \cdot \mathrm{FraAs}^{a}, \mathrm{~A} \cdot \mathrm{BArtl}^{b}, \mathrm{~W} \cdot \mathrm{Majerotto}^{c}$ \\ a Institut für Theoretische Physik, Universität Würzburg, Am Hubland, \\ D-97074 Würzburg, Germany \\ ${ }^{b}$ Institut für Theoretische Physik, Universität Wien, Boltzmanngasse 5, A-1090 Wien \\ ${ }^{c}$ Institut für Hochenergiephysik, Österreichische Akademie der Wissenschaften, \\ Nikolsdorfergasse 18, A-1050 Wien
}

\begin{abstract}
We study the production of charginos $e^{+} e^{-} \rightarrow \tilde{\chi}_{i}^{+} \tilde{\chi}_{j}^{-},(i, j=1,2)$ with polarized beams and the subsequent decays $\tilde{\chi}_{i}^{+} \rightarrow \tilde{\chi}_{k}^{0} \ell^{+} \nu_{\ell}$ and $\tilde{\chi}_{j}^{-} \rightarrow \tilde{\chi}_{l}^{0} \ell^{-} \bar{\nu}_{\ell},(k, l=1, \ldots, 4)$, including the complete spin correlations between production and decay. Analytical formulae are presented for the joint spin-density matrix of the charginos, for the chargino decay matrix and for the differential cross section of the combined processes of production and decays. We present numerical results for pair production of the lighter chargino with unpolarized beams and the leptonic decay of $\tilde{\chi}_{1}^{-}$into the lightest neutralino $\tilde{\chi}_{1}^{0}$. The lepton angular distribution and the forward-backward asymmetry are studied in four representative scenarios for $\sqrt{s}=192 \mathrm{GeV}$ and $\sqrt{s}=200 \mathrm{GeV}$.
\end{abstract}

${ }^{*}$ e-mail: gudi@physik.uni-wuerzburg.de 


\section{Introduction}

The search for supersymmetric particles is one of the main goals of LEP and of future $e^{+} e^{-}$ colliders. The charginos, the supersymmetric partners of the charged gauge and Higgs bosons, are of particular interest as they are expected to be lighter than the strongly interacting gluino and squarks. The lighter chargino $\tilde{\chi}_{1}^{ \pm}$could first be observed in experiments at $e^{+} e^{-}$colliders.

Most studies of chargino production $e^{+} e^{-} \rightarrow \tilde{\chi}_{i}^{+} \tilde{\chi}_{j}^{-}, i, j=1,2$, and chargino decays have been performed in the Minimal Supersymmetric Standard Model (MSSM). (See, for example [1, 2, 3], and references therein.) For a clear identification of charginos a precise analysis of their decay characteristics is indispensable. Angular distributions and angular correlations of the decay products of the charginos give valuable information on their gaugino and higgsino components and thus on the parameters of the MSSM.

Since angular distributions depend on the polarization of the parent particles one has to take into account the spin correlations between production and decay of the charginos. In general quantum mechanical interference effects between various polarization states of the decaying particles preclude simple factorization of the differential cross section into a production and a decay factor [4, 5], unless the production amplitude is dominated by a single spin component [6]. Recently, pair production and decay of the lighter chargino with spin correlations has also been studied in [7]. A variety of event generators for production and decay of SUSY particles, such as DFGT, SUSYGEN, GRACE and CompHEP \&, have been developed which partly also include spin correlations between production and decay.

In the present paper we study the process $e^{+} e^{-} \rightarrow \tilde{\chi}_{i}^{+} \tilde{\chi}_{j}^{-},(i, j=1,2)$ with polarized beams and the subsequent leptonic decays $\tilde{\chi}_{i}^{+} \rightarrow \tilde{\chi}_{k}^{0} \ell^{+} \nu_{\ell}, \tilde{\chi}_{j}^{-} \rightarrow \tilde{\chi}_{l}^{0} \ell^{-} \bar{\nu}_{\ell}$. The main purpose of this paper is the presentation of analytical formulae for the complete spin correlations between production and decay. The computation is done for complex couplings so that the formulae are also useful for the study of $\mathrm{CP}$ violating phenomena. The expression for the differential cross section is composed of the joint spin density matrix of the charginos and the decay matrices for their leptonic decay into one of the neutralino states.

The analytical expressions given for the production density matrix can also be used as a building block for processes with other chargino decay channels as e.g. hadronic decay or sequential chargino decay. Furthermore, the decay matrix can be combined with other chargino production processes as e.g. $e^{-} \gamma$ or $\gamma \gamma$ collisions.

Since in this article our emphasis is on the analytical formulae we restrict ourselves in the numerical calculations to the pair production of the lighter chargino with unpolarized beams, and to the leptonic decay of one chargino into the lightest neutralino $\tilde{\chi}_{1}^{0}$.

In sect. 2 we present the analytical expressions for the spin-density matrix for production, for the decay matrix and for the differential cross section. Numerical results for the lepton angular distribution for LEP 2 energies $\sqrt{s}=192 \mathrm{GeV}$ and $\sqrt{s}=200 \mathrm{GeV}$ are given in sect. 3 for four scenarios which differ significantly in the mixing character of the chargino and the lightest neutralino. 


\section{Analytical formulae}

\subsection{Definition of couplings}

We study the process

$e^{-}\left(p_{1}\right)+e^{+}\left(p_{2}\right) \rightarrow \tilde{\chi}_{i}^{+}\left(p_{3}\right)+\tilde{\chi}_{j}^{-}\left(p_{4}\right)$,

where the charginos decay leptonically:

$\tilde{\chi}_{i}^{+}\left(p_{3}\right) \rightarrow \tilde{\chi}_{k}^{0}\left(p_{5}\right)+\ell^{+}\left(p_{6}\right)+\nu\left(p_{7}\right)$,

and

$\tilde{\chi}_{j}^{-}\left(p_{4}\right) \rightarrow \tilde{\chi}_{l}^{0}\left(p_{8}\right)+\ell^{-}\left(p_{9}\right)+\bar{\nu}\left(p_{10}\right)$

The corresponding Feynman diagrams are shown in Fig. 1. The production process contains contributions from $\gamma$ - and $Z^{0}$-exchange in the direct channel and from $\tilde{\nu}$-exchange in the crossed channel. The decay process gets contributions from $W^{ \pm}, \tilde{e}_{L}$, and $\tilde{\nu}$ exchange in the different channels.

From the interaction Lagrangian of the MSSM ( in our notation and conventions we follow closely [9]):

$$
\begin{aligned}
& \mathcal{L}_{\gamma \tilde{\chi}_{i}^{+} \tilde{\chi}_{j}^{+}}=-e A_{\mu} \overline{\tilde{\chi}}_{i}^{+} \gamma^{\mu} \tilde{\chi}_{j}^{+} \delta_{i j}, \quad e>0, \\
& \mathcal{L}_{Z^{0} \tilde{\chi}_{i}^{+} \tilde{\chi}_{j}^{+}}=\frac{g}{\cos \theta_{W}} Z_{\mu} \overline{\tilde{\chi}}_{i}^{+} \gamma^{\mu}\left[O_{i j}^{L} P_{L}+O_{i j}^{\prime R} P_{R}\right] \tilde{\chi}_{j}^{+}, \\
& \mathcal{L}_{\ell \tilde{\nu} \tilde{\chi}_{i}^{+}}=-g U_{i 1}^{*} \overline{\tilde{\chi}}_{i}^{+} P_{L} \nu \tilde{\ell}_{L}^{*}-g V_{i 1}^{*} \overline{\tilde{\chi}}_{i}^{+C} P_{L} \ell \tilde{\nu}^{*}+\text { h.c. } \\
& \mathcal{L}_{W-\tilde{\chi}_{i}^{+} \tilde{\chi}_{k}^{0}}=g W_{\mu}^{-} \overline{\tilde{\chi}}_{k}^{0} \gamma^{\mu}\left[O_{k i}^{L} P_{L}+O_{k i}^{R} P_{R}\right] \tilde{\chi}_{i}^{+}+\text {h.c. } \\
& \mathcal{L}_{\ell \tilde{\tilde{\chi}_{k}^{0}}}=g f_{\ell k}^{L} \bar{\ell} P_{R} \tilde{\chi}_{k}^{0} \tilde{\ell}_{L}+g f_{\ell k}^{R} \bar{\ell} P_{L} \tilde{\chi}_{k}^{0} \tilde{\ell}_{R}+\text { h.c. }, \\
& \mathcal{L}_{\nu \tilde{\nu} \tilde{\chi}_{k}^{0}}=g f_{\nu k}^{L} \bar{\nu} P_{R} \tilde{\chi}_{k}^{0} \tilde{\nu}_{L}+\text { h.c. },
\end{aligned}
$$

one gets the couplings:

$$
\begin{aligned}
L_{\ell} & =T_{3 \ell}-e_{\ell} \sin ^{2} \theta_{W}, \quad R_{\ell}=-e_{\ell} \sin ^{2} \theta_{W} \\
f_{\ell k}^{L} & =-\sqrt{2}\left[\frac{1}{\cos \theta_{W}}\left(T_{3 \ell}-e_{\ell} \sin ^{2} \theta_{W}\right) N_{k 2}+e_{\ell} \sin \theta_{W} N_{k 1}\right] \\
f_{\ell k}^{R} & =-\sqrt{2} e_{\ell} \sin \theta_{W}\left[\tan \theta_{W} N_{k 2}^{*}-N_{k 1}^{*}\right] \\
f_{\nu k}^{L} & =-\sqrt{2} \frac{1}{\cos \theta_{W}} T_{3 \nu} N_{k 2} \\
O_{i j}^{\prime L} & =-V_{i 1} V_{j 1}^{*}-\frac{1}{2} V_{i 2} V_{j 2}^{*}+\delta_{i j} \sin ^{2} \Theta_{W}, \\
O_{i j}^{\prime R} & =-U_{i 1}^{*} U_{j 1}-\frac{1}{2} U_{i 2}^{*} U_{j 2}+\delta_{i j} \sin ^{2} \Theta_{W} \\
O_{k i}^{L} & =-1 / \sqrt{2}\left(\cos \beta N_{k 4}-\sin \beta N_{k 3}\right) V_{i 2}^{*}+\left(\sin \Theta_{W} N_{k 1}+\cos \Theta_{W} N_{k 2}\right) V_{i 1}^{*}, \\
O_{k i}^{R} & =+1 / \sqrt{2}\left(\sin \beta N_{k 4}^{*}+\cos \beta N_{k 3}^{*}\right) U_{i 2}+\left(\sin \Theta_{W} N_{k 1}^{*}+\cos \Theta_{W} N_{k 2}^{*}\right) U_{i 1},
\end{aligned}
$$


with $i, j=1,2$ and $k=1, \ldots, 4$. Here $P_{L, R}=\frac{1}{2}\left(1 \mp \gamma_{5}\right), g$ is the weak coupling constant $\left(g=e / \sin \theta_{W}\right)$, and $e_{\ell}$ and $T_{3 \ell}$ denote the charge and the third component of the weak isospin of the lepton $\ell$. Furthermore, $\tan \beta=\frac{v_{2}}{v_{1}}$ where $v_{1,2}$ are the vacuum expectation values of the two neutral Higgs fields, and $N_{m n}$ are the elements of the unitary $4 \times 4$ matrix which diagonalizes the neutral gaugino-higgsino mass matrix in the basis $\tilde{\gamma}, \tilde{Z}, \tilde{H}_{a}^{0}, \tilde{H}_{b}^{0}$. The chargino mass eigenstates $\tilde{\chi}_{i}^{+}=\left(\begin{array}{c}\chi_{i}^{+} \\ \chi_{i}^{-}\end{array}\right)$are defined by $\chi_{i}^{+}=V_{i 1} w^{+}+V_{i 2} h^{+}$and $\chi_{j}^{-}=$ $U_{j 1} w^{-}+U_{j 2} h^{-}$. Here $w^{ \pm}$and $h^{ \pm}$are the two-component spinor fields of the $\mathrm{W}$-ino and the charged higgsinos, respectively. Furthermore, $U_{m n}$ and $V_{m n}$ are the elements of the unitary $2 \times 2$ matrices which diagonalize the chargino mass matrix. For details see [1].

The helicity amplitudes $T_{P}^{\lambda_{i} \lambda_{j}}(\alpha)$ for production and $T_{D, \lambda_{i}}(\alpha), T_{D, \lambda_{j}}(\alpha)$ for the decays, corresponding to the Feynman diagrams in Fig. 1 are:

$$
\begin{aligned}
& T_{P}^{\lambda_{i} \lambda_{j}}(\gamma)=-\Delta(\gamma) \delta_{i j} \bar{v}\left(p_{2}\right) \gamma^{\mu} u\left(p_{1}\right) \bar{u}_{\lambda_{i}}\left(p_{3}\right) \gamma_{\mu} v_{\lambda_{j}}\left(p_{4}\right), \\
& T_{P}^{\lambda_{i} \lambda_{j}}(Z)=-\Delta(Z) \bar{v}\left(p_{2}\right) \gamma^{\mu}\left(L_{\ell} P_{L}+R_{\ell} P_{R}\right) u\left(p_{1}\right) \bar{u}_{\lambda_{i}}\left(p_{3}\right) \gamma_{\mu}\left(O_{i j}^{L} P_{L}+O_{i j}^{\prime R} P_{R}\right) v_{\lambda_{j}}\left(p_{4}\right), \\
& T_{P}^{\lambda_{i} \lambda_{j}}(\tilde{\nu})=-\Delta_{i j}(\tilde{\nu}) \bar{v}\left(p_{2}\right) P_{R} v_{\lambda_{i}}\left(p_{3}\right) \bar{u}_{\lambda_{j}}\left(p_{4}\right) P_{L} u\left(p_{1}\right), \\
& T_{D, \lambda_{i}}\left(W^{+}\right)=-\Delta(W) \bar{u}\left(p_{5}\right) \gamma^{\mu}\left(O_{k i}^{L} P_{L}+O_{k i}^{R} P_{R}\right) u_{\lambda_{i}}\left(p_{3}\right) \bar{u}\left(p_{7}\right) \gamma_{\mu} P_{L} v\left(p_{6}\right), \\
& T_{D, \lambda_{i}}\left(\tilde{\ell}_{L}\right)=-\Delta_{i}(\tilde{\ell} L) \bar{u}\left(p_{7}\right) P_{R} u_{\lambda_{i}}\left(p_{3}\right) \bar{u}\left(p_{5}\right) P_{L} v\left(p_{6}\right) \\
& T_{D, \lambda_{i}}(\tilde{\nu})=+\Delta_{i}(\tilde{\nu}) \bar{u}\left(p_{6}\right) P_{L} u_{\lambda_{i}}\left(p_{3}\right) \bar{u}\left(p_{5}\right) P_{R} v\left(p_{7}\right) \\
& T_{D, \lambda_{j}}\left(W^{-}\right)=-\Delta(W) \bar{u}\left(p_{8}\right) \gamma^{\mu}\left(O_{l j}^{L *} P_{R}+O_{l j}^{R *} P_{L}\right) u_{\lambda_{j}}\left(p_{4}\right) \bar{u}\left(p_{10}\right) \gamma_{\mu} P_{R} v\left(p_{9}\right) \\
& T_{D, \lambda_{j}}\left(\tilde{\ell}_{L}\right)=-\Delta_{j}\left(\tilde{\ell}_{L}\right) \bar{u}\left(p_{10}\right) P_{L} u_{\lambda_{j}}\left(p_{4}\right) \bar{u}\left(p_{8}\right) P_{R} v\left(p_{9}\right) \\
& T_{D, \lambda_{j}}(\tilde{\nu})=+\Delta_{j}(\tilde{\nu}) \bar{u}\left(p_{9}\right) P_{R} u_{\lambda_{j}}\left(p_{4}\right) \bar{u}\left(p_{8}\right) P_{L} v\left(p_{10}\right) .
\end{aligned}
$$

In the following the indices of the couplings $O_{i j}^{L L, R}$ and $O_{k i}^{L, R}, O_{l j}^{L, R}$ are suppressed. In eqs. (14)-(22) the propagators $\Delta(\gamma)$ etc. include all couplings apart from $O^{L, R}$ and $O^{\prime} L, R$. They are defined as follows:

$$
\begin{aligned}
& \Delta(\gamma)=\frac{i e^{2}}{k^{2}}, \quad \Delta(Z)=\frac{g^{2}}{\cos \Theta_{W}^{2}} \frac{i}{k^{2}-m_{Z}^{2}+i m_{Z} \Gamma_{Z}}, \quad \Delta_{i j}(\tilde{\nu})=\frac{i g^{2} V_{i 1} V_{j 1}^{*}}{k^{2}-m_{\tilde{\nu}}^{2}+i m_{\tilde{\nu}} \Gamma_{\tilde{\nu}}} \\
& \Delta(W)=\frac{g^{2}}{\sqrt{2}} \frac{i}{k^{2}-m_{W}^{2}+i m_{W} \Gamma_{W}}, \\
& \Delta_{i}\left(\tilde{\ell}_{L}\right)=\frac{i g^{2} U_{i 1} f_{\ell k}^{* L}}{k^{2}-m_{\tilde{\ell}_{L}}^{2}+i m_{\tilde{\ell}_{L}} \Gamma_{\tilde{\ell}_{L}}}, \quad \Delta_{i}(\tilde{\nu})=\frac{i g^{2} V_{i 1}^{*} f_{\nu k}^{L}}{k^{2}-m_{\tilde{\nu}}^{2}+i m_{\tilde{\nu}} \Gamma_{\tilde{\nu}}} \\
& \Delta_{j}\left(\tilde{\ell}_{L}\right)=\frac{i g^{2} U_{j 1}^{*} f_{\ell l}^{L}}{k^{2}-m_{\tilde{\ell}_{L}}^{2}+i m_{\tilde{\ell}_{L}} \Gamma_{\tilde{\ell}_{L}}}, \quad \Delta_{j}(\tilde{\nu})=\frac{i g^{2} V_{j 1} f_{\nu l}^{* L}}{k^{2}-m_{\tilde{\nu}}^{2}+i m_{\tilde{\nu}} \Gamma_{\tilde{\nu}}} .
\end{aligned}
$$

Here $k^{2}$ denotes the four-momentum squared of the respective particle.

For the calculation of the amplitude of the combined processes of production and decays, eqs.(11) - (3), we use the same formalism that we already adopted for the analogous 
production of neutralinos and their decays with polarized beams [10, 11] following the method of [12]. The amplitude for the whole process is

$T=\Delta\left(\tilde{\chi}_{i}^{+}\right) \Delta\left(\tilde{\chi}_{j}^{-}\right) \sum_{\lambda_{i}, \lambda_{j}} T_{P}^{\lambda_{i} \lambda_{j}} T_{D, \lambda_{i} \lambda_{j}}$

with the helicity amplitude for the production process $T_{P}^{\lambda_{i} \lambda_{j}}$ and that for the decay processes $T_{D, \lambda_{i} \lambda_{j}}=T_{D, \lambda_{i}} T_{D, \lambda_{j}}$, and the propagators $\Delta\left(\tilde{\chi}_{i, j}^{ \pm}\right)=1 /\left[s_{i, j}-m_{i, j}^{2}+i m_{i, j} \Gamma_{i, j}\right]$. Here $\lambda_{i, j}, s_{i, j}, m_{i, j}, \Gamma_{i, j}$ denote the helicity, four-momentum squared, mass and width of $\tilde{\chi}_{i, j}^{ \pm}$. The amplitude squared

$|T|^{2}=\left|\Delta\left(\tilde{\chi}_{i}^{+}\right)\right|^{2}\left|\Delta\left(\tilde{\chi}_{j}^{-}\right)\right|^{2} \rho^{P, \lambda_{i} \lambda_{j} \lambda_{i}^{\prime} \lambda_{j}^{\prime}} \rho_{\lambda_{i}^{\prime} \lambda_{i}}^{D} \rho_{\lambda_{j}^{\prime} \lambda_{j}}^{D} \quad$ (sum convention used)

is thus composed of the (unnormalized) spin density production matrix

$\rho^{P, \lambda_{i} \lambda_{j} \lambda_{i}^{\prime} \lambda_{j}^{\prime}}=T_{P}^{\lambda_{i} \lambda_{j}} T_{P}^{\lambda_{i}^{\prime} \lambda_{j}^{\prime} *}$

of $\tilde{\chi}_{i, j}^{ \pm}$and the decay matrices

$\rho_{\lambda_{i}^{\prime} \lambda_{i}}^{D}=T_{D, \lambda_{i}} T_{D, \lambda_{i}^{\prime}}^{*} \quad$ and $\quad \rho_{\lambda_{j}^{\prime} \lambda_{j}}^{D}=T_{D, \lambda_{j}} T_{D, \lambda_{j}^{\prime}}^{*}$.

Interference terms between various helicity amplitudes preclude factorization in a production factor $\sum_{\lambda_{i} \lambda_{j}}\left|T_{P}^{\lambda_{i} \lambda_{j}}\right|^{2}$ times a decay factor $\bar{\sum}_{\lambda_{i} \lambda_{j}}\left|T_{D, \lambda_{i} \lambda_{j}}\right|^{2}$.

The differential cross section in the laboratory system is then given by:

$d \sigma=\frac{1}{8 E_{b}^{2}}|T|^{2}(2 \pi)^{4} \delta^{4}\left(p_{1}+p_{2}-\sum_{i} p_{i}\right) d \operatorname{lips}\left(p_{3} \ldots p_{10}\right)$,

$E_{b}$ denotes the beam energy and $d \operatorname{lips}\left(p_{3}, \ldots, p_{10}\right)=\prod_{i} \frac{d^{3} p_{i}}{(2 \pi)^{3} 2 p_{i}^{0}} \quad, i=3, \ldots, 10$.

\subsection{Spin density production matrix}

We use the general formalism to calculate the helicity amplitudes for production and decay of a particle with four-momentum $p$ and mass $m$. For this purpose we introduce three spacelike four-vectors $s_{\mu}^{a},(a=1,2,3)$, the spin vectors, which together with $p / m$ form an orthonormal set 12]:

$\frac{p}{m} \cdot s^{a}=0$

$s^{a} \cdot s^{a^{\prime}}=-\delta^{a a^{\prime}}$,

$s_{\mu}^{a} \cdot s_{\nu}^{a}=-g_{\mu \nu}+\frac{p_{\mu} p_{\nu}}{m^{2}}$, summed over a.

In computing the density matrices for production and decay one makes use of the BouchiatMichel formulae [12]:

$$
\begin{aligned}
& u_{\lambda^{\prime}}(p) \bar{u}_{\lambda}(p)=\frac{1}{2}\left[\delta_{\lambda \lambda^{\prime}}+\gamma_{5} \not \sigma_{\lambda \lambda^{\prime}}^{a} \sigma^{a}[\not p+m),\right. \\
& v_{\lambda^{\prime}}(p) \bar{v}_{\lambda}(p)=\frac{1}{2}\left[\delta_{\lambda^{\prime} \lambda}+\gamma_{5} \phi^{a} \sigma_{\lambda^{\prime} \lambda}^{a}\right](\not p-m) .
\end{aligned}
$$




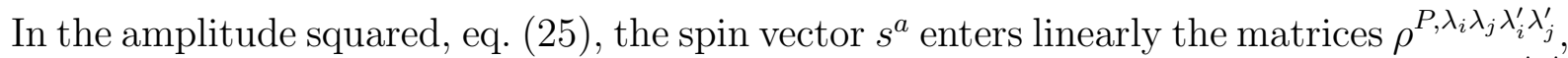
$\rho_{\lambda_{i}^{\prime} \lambda_{i}}^{D}$ and $\rho_{\lambda_{j}^{\prime} \lambda_{j}}^{D}$. It is appropriate to separate the spin density production matrix $\rho^{P, \lambda_{i} \lambda_{j} \lambda_{i}^{\prime} \lambda_{j}^{\prime}}$ into four parts:

$\rho^{P, \lambda_{i} \lambda_{j} \lambda_{i}^{\prime} \lambda_{j}^{\prime}}=\sum_{\alpha \beta}\left(P^{\lambda_{i} \lambda_{j} \lambda_{i}^{\prime} \lambda_{j}^{\prime}}(\alpha \beta)+\Sigma_{a}^{P, \lambda_{i} \lambda_{j} \lambda_{i}^{\prime} \lambda_{j}^{\prime}}(\alpha \beta)+\Sigma_{b}^{P, \lambda_{i} \lambda_{j} \lambda_{i}^{\prime} \lambda_{j}^{\prime}}(\alpha \beta)+\Sigma_{a b}^{P, \lambda_{i} \lambda_{j} \lambda_{i}^{\prime} \lambda_{j}^{\prime}}(\alpha \beta)\right)$,

with $(\alpha \beta)=(\gamma \gamma),\left(\gamma Z^{0}\right),(\gamma \tilde{\nu}),\left(Z^{0} Z^{0}\right),\left(Z^{0} \tilde{\nu}\right),(\tilde{\nu} \tilde{\nu})$ denoting the exchanged particles, where the second argument corresponds to the complex conjugated amplitude. One has only these six combinations of exchanged particles in eq.(34) due to the relation:

$\rho^{P, \lambda_{i} \lambda_{j} \lambda_{i}^{\prime} \lambda_{j}^{\prime}}(\alpha \beta)=\left(\rho^{P, \lambda_{i} \lambda_{j} \lambda_{i}^{\prime} \lambda_{j}^{\prime}}(\beta \alpha)\right)^{*}$.

The four parts are defined as follows:

$$
P^{\lambda_{i} \lambda_{j} \lambda_{i}^{\prime} \lambda_{j}^{\prime}}(\alpha \beta)=\delta_{\lambda_{i} \lambda_{i}^{\prime}} \delta_{\lambda_{j} \lambda_{j}^{\prime}} P(\alpha \beta),
$$

which is independent of the polarization of the charginos,

$\Sigma_{a}^{P, \lambda_{i} \lambda_{j} \lambda_{i}^{\prime} \lambda_{j}^{\prime}}(\alpha \beta)=\delta_{\lambda_{j} \lambda_{j}^{\prime}} \sigma_{\lambda_{i} \lambda_{i}^{\prime}}^{a} \Sigma_{a}^{P}(\alpha \beta)$,

where only the polarization vector $s^{a}$ of the chargino $\tilde{\chi}_{i}^{+}\left(p_{3}\right)$ contributes,

$\Sigma_{b}^{P, \lambda_{i} \lambda_{i}^{\prime} \lambda_{j} \lambda_{j}^{\prime}}(\alpha \beta)=\delta_{\lambda_{i} \lambda_{i}^{\prime}} \sigma_{\lambda_{j} \lambda_{j}^{\prime}}^{b} \Sigma_{b}^{P}(\alpha \beta)$,

where only the polarization vector $s^{b}$ of the chargino $\tilde{\chi}_{j}^{-}\left(p_{4}\right)$ contributes,

$\Sigma_{a b}^{P \lambda_{i} \lambda_{j} \lambda_{i}^{\prime} \lambda_{j}^{\prime}}(\alpha \beta)=\sigma_{\lambda_{i} \lambda_{i}^{\prime}}^{a} \sigma_{\lambda_{j} \lambda_{j}^{\prime}}^{b} \Sigma_{a b}^{P}(\alpha \beta)$,

which includes the polarization vectors $s^{a}$ and $s^{b}$ of both charginos $\tilde{\chi}_{i}^{+}$and $\tilde{\chi}_{j}^{-}$. $\sigma_{\lambda_{i} \lambda_{i}^{\prime}}^{a}, \sigma_{\lambda_{j} \lambda_{j}^{\prime}}^{b}$ are the $2 \times 2$ Pauli matrices.

In the following we give the analytical formulae for the various quantities in eq.(34). Scalar products are denoted by $\left(p_{i} p_{j}\right)$. We use the abbrevation $\left[p_{i} p_{j} p_{k} p_{l}\right]=i \epsilon_{\mu \nu \rho \sigma} p_{i}^{\mu} p_{j}^{\nu} p_{k}^{\rho} p_{l}^{\sigma}$, where the totally antisymmetric tensor $\epsilon_{\mu \nu \rho \sigma}=+1$, if $\{\mu \nu \rho \sigma\}$ is an even permutation of $\{0,1,2,3\}$.

It is useful to define a coupling $c^{L(R)}(\alpha)$ for the exchanged particle $(\alpha)$ with

$$
\begin{aligned}
& c^{L}(\gamma)=1 \\
& c^{L}\left(Z^{0}\right)=L_{\ell} \\
& c^{L}(\tilde{\nu})=1 \\
& c^{R}(\gamma)=1 \\
& c^{R}\left(Z^{0}\right)=R_{\ell} \\
& c^{R}(\tilde{\nu})=0
\end{aligned}
$$

We further introduce the combination $c_{ \pm}^{P}(\alpha \beta)$ which combines the coupling $c^{L(R)}(\alpha)$ with the longitudinal beam polarization $P_{1}^{3}$ and $P_{2}^{3}$ of the incoming particles $e^{-}\left(p_{1}\right)$ and $e^{+}\left(p_{2}\right)$ :

$c_{ \pm}^{P}(\alpha \beta)= \pm c^{L}(\alpha) c^{L}(\beta)\left(1-P_{1}^{3}\right)\left(1+P_{2}^{3}\right)+c^{R}(\alpha) c^{R}(\beta)\left(1+P_{1}^{3}\right)\left(1-P_{2}^{3}\right)$

For unpolarized beams one has $P_{1}^{3}=0=P_{2}^{3}$ in eq.(46). 


\subsection{1 $P(\alpha \beta)$}

This is the part of eq.(34) which is independent of the chargino polarization vectors. In $P(\alpha \beta)$ only three different pairs of scalar products contribute:

$f_{1}=\left(p_{1} p_{4}\right)\left(p_{2} p_{3}\right)$,

$f_{2}=\left(p_{1} p_{3}\right)\left(p_{2} p_{4}\right)$,

$f_{3}=m_{i} m_{j}\left(p_{1} p_{2}\right)$.

The analytical expressions for $P(\alpha \beta)$, eq. (36), read:

$$
\begin{aligned}
P(\gamma \gamma)= & |\Delta(\gamma)|^{2} c_{+}^{P}(\gamma \gamma) \delta_{i j}\left(f_{1}+f_{2}+f_{3}\right), \\
P(\gamma Z)= & 2 \operatorname{Re}\left\{\Delta ( \gamma ) \Delta ( Z ) ^ { * } \delta _ { i j } \frac { 1 } { 2 } \left[c_{+}^{P}(\gamma Z)\left(O^{\prime L *}+O^{\prime R *}\right)\left(f_{1}+f_{2}+f_{3}\right)\right.\right. \\
& \left.\left.+c_{-}^{P}(\gamma Z)\left(O^{\prime R *}-O^{\prime}{ }^{*}\right)\left(f_{1}-f_{2}\right)\right]\right\} \\
P(\gamma \tilde{\nu})= & 2 \operatorname{Re}\left\{\Delta(\gamma) \Delta_{i j}^{*}(\tilde{\nu}) \delta_{i j} \frac{1}{4} c_{+}^{P}(\gamma \tilde{\nu})\left(2 f_{1}+f_{3}\right)\right\} \\
P(Z Z)= & |\Delta(Z)|^{2} \frac{1}{2}\left[c _ { + } ^ { P } ( Z Z ) \left(\left(\left|O^{\prime L}\right|^{2}+\left|O^{\prime}\right|^{2}\right)\left(f_{1}+f_{2}\right)\right.\right. \\
& \left.\left.-\left(O^{L} O^{\prime R *}+O^{R} O^{\prime} L *\right)\left(-f_{3}\right)\right)+c_{-}^{P}(Z Z)\left(\left|O^{\prime R}\right|^{2}-\left|O^{\prime}\right|^{2}\right)\left(f_{1}-f_{2}\right)\right], \\
P(Z \tilde{\nu})= & 2 \operatorname{Re}\left\{\Delta(Z) \Delta_{i j}^{*}(\tilde{\nu}) \frac{1}{4} c_{+}^{P}(Z \tilde{\nu})\left(2 O^{\prime L} f_{1}+O^{\prime} f_{3}\right)\right\} \\
P(\tilde{\nu} \tilde{\nu})= & \left|\Delta_{i j}(\tilde{\nu})\right|^{2} \frac{1}{4} c_{+}^{P}(\tilde{\nu} \tilde{\nu}) f_{1} .
\end{aligned}
$$

\subsection{2 $\Sigma_{a}^{P}(\alpha \beta)$ and $\Sigma_{b}^{P}(\alpha \beta)$}

This is the part of eq.(34) which contains only one polarization vector, either $s^{a}$ of $\tilde{\chi}_{i}^{+}$or $s^{b}$ of $\tilde{\chi}_{j}^{-}$. In $\Sigma_{a}^{P}(\alpha \beta)$, eq. (37), the following five products with the polarization vector $s^{a}$ appear:

$$
\begin{aligned}
& f_{1}^{a}=m_{i}\left(p_{2} p_{4}\right)\left(p_{1} s^{a}\right), \\
& f_{2}^{a}=m_{i}\left(p_{1} p_{4}\right)\left(p_{2} s^{a}\right), \\
& f_{3}^{a}=m_{j}\left(p_{2} p_{3}\right)\left(p_{1} s^{a}\right), \\
& f_{4}^{a}=m_{j}\left(p_{1} p_{3}\right)\left(p_{2} s^{a}\right), \\
& f_{5}^{a}=m_{j}\left[p_{2} p_{1} s^{a} p_{3}\right] .
\end{aligned}
$$

The contributions $\sum_{a}^{P}(\alpha \beta)$ in eq. 37$)$ are:

$$
\begin{aligned}
\Sigma_{a}^{P}(\gamma \gamma)= & |\Delta(\gamma)|^{2} c_{-}^{P}(\gamma \gamma) \delta_{i j}\left(-f_{1}^{a}+f_{2}^{a}-f_{3}^{a}+f_{4}^{a}\right) \\
\Sigma_{a}^{P}(\gamma Z)= & 2 \operatorname{Re}\left\{\Delta ( \gamma ) \Delta ^ { * } ( Z ) \frac { 1 } { 2 } \delta _ { i j } \left[c_{+}^{P}(\gamma Z)\left(O^{\prime R *}-O^{\prime L *}\right)\left(f_{1}^{a}+f_{2}^{a}\right)+c_{-}^{P}(\gamma Z)\right.\right. \\
& \left.\left.\left(\left(O^{\prime} R *+O^{\prime L *}\right)\left(-f_{1}^{a}+f_{2}^{a}-f_{3}^{a}+f_{4}^{a}\right)-\left(O^{\prime R *}-O^{\prime L *}\right) f_{5}^{a}\right)\right]\right\}
\end{aligned}
$$




$$
\begin{aligned}
& \Sigma_{a}^{P}(\gamma \tilde{\nu})=2 \operatorname{Re}\left\{\Delta(\gamma) \Delta_{i j}^{*}(\tilde{\nu}) \frac{1}{4} \delta_{i j} c_{+}^{P}(\gamma \tilde{\nu})\left(-2 f_{2}^{a}+f_{3}^{a}-f_{4}^{a}-f_{5}^{a}\right)\right\} \\
& \Sigma_{a}^{P}(Z Z)=|\Delta(Z)|^{2} \frac{1}{2}\left[c_{+}^{P}(Z Z)\left(\left|O^{\prime} R\right|^{2}-\left|O^{\prime L}\right|^{2}\right)\left(f_{1}^{a}+f_{2}^{a}\right)\right. \\
& +c_{-}^{P}(Z Z)\left(\left(O^{\prime L} O^{\prime R *}+O^{\prime} R O^{\prime L *}\right)\left(-f_{3}^{a}+f_{4}^{a}\right)\right. \\
& \left.\left.+\left(\left|O^{\prime} R\right|^{2}+\left|O^{\prime} L\right|^{2}\right)\left(-f_{1}^{a}+f_{2}^{a}\right)-\left(O^{\prime} L O^{\prime R *}-O^{\prime} R O^{\prime} L *\right) f_{5}^{a}\right)\right], \\
& \Sigma_{a}^{P}(Z \tilde{\nu})=2 \operatorname{Re}\left\{\Delta(Z) \Delta_{i j}^{*}(\tilde{\nu}) \frac{1}{4} c_{+}^{P}(Z \tilde{\nu})\left(-2 O^{\prime L} f_{2}^{a}-O^{\prime R}\left(-f_{3}^{a}+f_{4}^{a}+f_{5}^{a}\right)\right)\right\}, \\
& \Sigma_{a}^{P}(\tilde{\nu} \tilde{\nu})=\left|\Delta_{i j}(\tilde{\nu})\right|^{2} \frac{1}{4} c_{+}^{P}(\tilde{\nu} \tilde{\nu})\left(-f_{2}^{a}\right) .
\end{aligned}
$$

In the analogous formulae for $\sum_{b}^{P}(\alpha \beta)$, eq. (38), the following five products containing the polarization vector $s^{b}$ contribute:

$$
\begin{aligned}
f_{1}^{b} & =m_{i}\left(p_{2} p_{4}\right)\left(p_{1} s^{b}\right), \\
f_{2}^{b} & =m_{i}\left(p_{1} p_{4}\right)\left(p_{2} s^{b}\right), \\
f_{3}^{b} & =m_{j}\left(p_{2} p_{3}\right)\left(p_{1} s^{b}\right), \\
f_{4}^{b} & =m_{j}\left(p_{1} p_{3}\right)\left(p_{2} s^{b}\right), \\
f_{5}^{b} & =m_{i}\left[p_{2} p_{1} s^{b} p_{4}\right],
\end{aligned}
$$

The corresponding formulae for $\sum_{b}^{P}(\alpha \beta)$ are obtained by substituting in eqs.(61)-(66):

$f_{1}^{a} \rightarrow-f_{4}^{b}, \quad f_{2}^{a} \rightarrow-f_{3}^{b}, \quad f_{3}^{a} \rightarrow-f_{2}^{b}, \quad f_{4}^{a} \rightarrow-f_{1}^{b}, \quad f_{5}^{a} \rightarrow-f_{5}^{b}$.

\subsection{3 $\Sigma_{a b}^{P}(\alpha \beta)$}

This is the part of eq. (34) which contains both polarization vectors $s^{a}$ of $\tilde{\chi}_{i}^{+}$and $s^{b}$ of $\tilde{\chi}_{j}^{-}$. It can be expressed by eight different combinations of products with both spin vectors:

$$
\begin{aligned}
& f_{1}^{a b}=\left(p_{3} p_{4}\right)\left(p_{1} s^{a}\right)\left(p_{2} s^{b}\right), \\
& f_{2}^{a b}=m_{i} m_{j}\left(p_{1} s^{a}\right)\left(p_{2} s^{b}\right), \\
& f_{3}^{a b}=\left(p_{3} p_{4}\right)\left(p_{1} s^{b}\right)\left(p_{2} s^{a}\right), \\
& f_{4}^{a b}=m_{i} m_{j}\left(p_{1} s^{b}\right)\left(p_{2} s^{a}\right), \\
& f_{5}^{a b}=\left(s^{a} s^{b}\right)\left[\left(p_{1} p_{4}\right)\left(p_{2} p_{3}\right)-\left(p_{1} p_{2}\right)\left(p_{3} p_{4}\right)+\left(p_{1} p_{3}\right)\left(p_{2} p_{4}\right)\right], \\
& f_{6}^{a b}=\left(p_{3} s^{b}\right)\left[\left(p_{1} p_{2}\right)\left(p_{4} s^{a}\right)-\left(p_{1} p_{4}\right)\left(p_{2} s^{a}\right)-\left(p_{2} p_{4}\right)\left(p_{1} s^{a}\right)\right], \\
& f_{7}^{a b}=\left(p_{4} s^{a}\right)\left[\left(p_{1} p_{3}\right)\left(p_{2} s^{b}\right)+\left(p_{2} p_{3}\right)\left(p_{1} s^{b}\right)\right], \\
& f_{8}^{a b}=\left(p_{2} p_{4}\right)\left[s^{b} s^{a} p_{3} p_{1}\right]-\left(p_{3} p_{1}\right)\left[s^{b} s^{a} p_{2} p_{4}\right]+\left(p_{2} s^{b}\right)\left[s^{a} p_{3} p_{1} p_{4}\right]+\left(s^{a} p_{1}\right)\left[s^{b} p_{3} p_{2} p_{4}\right] .
\end{aligned}
$$

The contributions $\sum_{a b}^{P}(\alpha \beta)$ in eq. (39) are:

$$
\begin{aligned}
\Sigma_{a b}^{P}(\gamma \gamma) & =|\Delta(\gamma)|^{2} c_{+}^{P}(\gamma \gamma) \delta_{i j}\left(-f_{1}^{a b}-f_{2}^{a b}-f_{3}^{a b}-f_{4}^{a b}-f_{5}^{a b}-f_{6}^{a b}+f_{7}^{a b}\right) \\
\Sigma_{a b}^{P}(\gamma Z) & =2 \operatorname{Re}\left\{\Delta ( \gamma ) \Delta ^ { * } ( Z ) \frac { 1 } { 2 } \delta _ { i j } \left[c _ { + } ^ { P } ( \gamma Z ) \left(\left(O^{\prime R *}-O^{\prime L *}\right) f_{8}^{a b}\right.\right.\right.
\end{aligned}
$$




$$
\begin{aligned}
& \left.+\left(O^{\prime R *}+O^{\prime L *}\right)\left(-f_{1}^{a b}-f_{2}^{a b}-f_{3}^{a b}-f_{4}^{a b}-f_{5}^{a b}-f_{6}^{a b}+f_{7}^{a b}\right)\right) \\
& \left.\left.+c_{-}^{P}(\gamma Z)\left(O^{\prime R *}-O^{\prime L *}\right)\left(f_{2}^{a b}-f_{4}^{a b}\right)\right]\right\} \\
\Sigma_{a b}^{P}(\gamma \tilde{\nu})= & 2 R e\left\{\Delta(\gamma) \Delta_{i j}^{*}(\tilde{\nu}) \frac{1}{4} \delta_{i j} c_{+}^{P}(\gamma \tilde{\nu})\left(-f_{1}^{a b}-f_{3}^{a b}-2 f_{4}^{a b}-f_{5}^{a b}-f_{6}^{a b}+f_{7}^{a b}-f_{8}^{a b}\right)\right\} \\
\Sigma_{a b}^{P}(Z Z)= & |\Delta(Z)|^{2} \frac{1}{2}\left[c _ { + } ^ { P } ( Z Z ) \left(\left(O^{\prime L} O^{\prime R *}+O^{\prime} R O^{\prime L *}\right)\right.\right. \\
& \left(-f_{1}^{a b}-f_{3}^{a b}-f_{5}^{a b}-f_{6}^{a b}+f_{7}^{a b}\right)+\left(\left|O^{\prime} R\right|^{2}+\left|O^{\prime L}\right|^{2}\right)\left(-f_{2}^{a b}-f_{4}^{a b}\right) \\
& \left.-\left(O^{\prime} L O^{\prime} R *-O^{\prime} R O^{\prime L *}\right)\left(-f_{8}^{a b}\right)\right) \\
& \left.+c_{-}^{P}(Z Z)\left(\left|O^{\prime}\right|^{2}-\left|O^{\prime} R\right|^{2}\right)\left(-f_{2}^{a b}+f_{4}^{a b}\right)\right] \\
\Sigma_{a b}^{P}(Z \tilde{\nu})= & 2 R e\left\{\Delta ( Z ) \Delta _ { i j } ^ { * } ( \tilde { \nu } ) \frac { 1 } { 4 } c _ { + } ^ { P } ( Z \tilde { \nu } ) \left(-2 O^{\prime L} f_{4}^{a b}\right.\right. \\
& \left.\left.-O^{\prime} R\left(f_{1}^{a b}+f_{3}^{a b}+f_{5}^{a b}+f_{6}^{a b}-f_{7}^{a b}+f_{8}^{a b}\right)\right)\right\} \\
\Sigma_{a b}^{P}(\tilde{\nu} \tilde{\nu})= & \left|\Delta_{i j}(\tilde{\nu})\right|^{2} \frac{1}{4} c_{+}^{P}(\tilde{\nu} \tilde{\nu})\left(-f_{4}^{a b}\right)
\end{aligned}
$$

\subsection{Decay matrix}

In the following we give the analytical formulae for the (unnormalized) decay matrices for both decays $\tilde{\chi}_{i}^{+}\left(p_{3}\right) \rightarrow \tilde{\chi}_{k}^{0}\left(p_{5}\right)+\ell^{+}\left(p_{6}\right)+\nu\left(p_{7}\right)$ and $\tilde{\chi}_{j}^{-}\left(p_{4}\right) \rightarrow \tilde{\chi}_{l}^{0}\left(p_{8}\right)+\ell^{-}\left(p_{9}\right)+\bar{\nu}\left(p_{10}\right)$ (see Fig. 1). In the following $m_{k}$ and $m_{l}$ denote the masses of the neutralinos $\tilde{\chi}_{k}^{0}$ and $\tilde{\chi}_{l}^{0}$. Analogously to eq. (34) the decay matrices $\rho_{\lambda_{i}^{\prime} \lambda_{i}}^{D}$ and $\rho_{\lambda_{j}^{\prime} \lambda_{j}}^{D}$ can both be separated into two parts:

$$
\begin{aligned}
& \rho_{\lambda_{i}^{\prime} \lambda_{i}}^{D}=\sum_{\alpha \beta}\left(D_{\lambda_{i}^{\prime} \lambda_{i}}(\alpha \beta)+\Sigma_{a, \lambda_{i}^{\prime} \lambda_{i}}^{D}(\alpha \beta)\right), \\
& \rho_{\lambda_{j}^{\prime} \lambda_{j}}^{D}=\sum_{\alpha \beta}\left(D_{\lambda_{j}^{\prime} \lambda_{j}}(\alpha \beta)+\Sigma_{b, \lambda_{j}^{\prime} \lambda_{j}}^{D}(\alpha \beta)\right),
\end{aligned}
$$

with $(\alpha \beta)=(W W),\left(W \tilde{\ell}_{L}\right),(W \tilde{\nu}),\left(\tilde{\ell}_{L} \tilde{\ell}_{L}\right),\left(\tilde{\ell}_{L} \tilde{\nu}\right),(\tilde{\nu} \tilde{\nu})$ denoting the exchanged particle. The second argument corresponds to the complex conjugated amplitude. Here we have also used the relation analogous to eq.(35). The combinations $(\beta \alpha)$ are therefore already included.

The two parts in eqs. 87) and (88) are given as follows:

$$
D_{\lambda_{i}^{\prime} \lambda_{i}}(\alpha \beta)=\delta_{\lambda_{i}^{\prime} \lambda_{i}} D_{i}(\alpha \beta)
$$

which is independent of the polarization vector of the chargino $\tilde{\chi}_{i}^{+}\left(p_{3}\right)$,

$$
\Sigma_{a, \lambda_{i}^{\prime} \lambda_{i}}^{D}(\alpha \beta)=\sigma_{\lambda_{i}^{\prime} \lambda_{i}}^{a} \Sigma_{a}^{D}(\alpha \beta),
$$

where the polarization vector $s^{a}$ of the chargino $\tilde{\chi}_{i}^{+}\left(p_{3}\right)$ contributes,

$$
D_{\lambda_{j}^{\prime} \lambda_{j}}(\alpha \beta)=\delta_{\lambda_{j}^{\prime} \lambda_{j}} D_{j}(\alpha \beta)
$$

which is independent of the polarization vector of the chargino $\tilde{\chi}_{j}^{-}\left(p_{4}\right)$, 


$$
\Sigma_{b, \lambda_{j}^{\prime} \lambda_{j}}^{D}(\alpha \beta)=\sigma_{\lambda_{j}^{\prime} \lambda_{j}}^{b} \Sigma_{b}^{D}(\alpha \beta),
$$

where the polarization vector $s^{b}$ of the chargino $\tilde{\chi}_{j}^{-}\left(p_{4}\right)$ contributes.

\subsection{1 $\quad D_{i}(\alpha \beta)$ and $D_{j}(\alpha \beta)$}

These are the parts of eq.(87) and eq. (88) which are independent of the polarization vector $s^{a}\left(s^{b}\right)$ of the decaying chargino $\tilde{\chi}_{i}^{+}\left(\tilde{\chi}_{j}^{-}\right)$. In the term $D_{i}(\alpha \beta)$, eq.(89), for the decay of $\tilde{\chi}_{i}^{+}\left(p_{3}\right)$ only three different pairs of scalar products contribute:

$$
\begin{aligned}
& g_{1}=\left(p_{5} p_{7}\right)\left(p_{3} p_{6}\right), \\
& g_{2}=\left(p_{5} p_{6}\right)\left(p_{3} p_{7}\right), \\
& g_{3}=\left(m_{i} m_{k}\right)\left(p_{6} p_{7}\right) .
\end{aligned}
$$

The analytical expressions for $D_{i}(\alpha \beta)$, eq.(89), read:

$$
\begin{aligned}
D_{i}\left(W^{+} W^{+}\right)= & |\Delta(W)|^{2} 4\left[\left(\left|O^{L}\right|^{2}+\left|O^{R}\right|^{2}\right)\left(g_{1}+g_{2}\right)-\left(O^{L *} O^{R}+O^{L} O^{R *}\right) g_{3}\right. \\
& \left.-\left(\left|O^{R}\right|^{2}-\left|O^{L}\right|^{2}\right)\left(g_{1}-g_{2}\right)\right], \\
D_{i}\left(W^{+} \tilde{\ell}_{L}\right)= & 2 \operatorname{Re}\left\{\Delta(W) \Delta_{i}^{*}\left(\tilde{\ell}_{L}\right) 2\left(2 O^{R} g_{2}-O^{L} g_{3}\right)\right\} \\
D_{i}\left(W^{+} \tilde{\nu}\right)= & 2 \operatorname{Re}\left\{-\Delta(W) \Delta_{i}^{*}(\tilde{\nu}) 2\left(2 O^{L} g_{1}-O^{R} g_{3}\right)\right\} \\
D_{i}\left(\tilde{\ell}_{L} \tilde{\ell}_{L}\right)= & \left|\Delta_{i}\left(\tilde{\ell}_{L}\right)\right|^{2} 2 g_{2}, \\
D_{i}\left(\tilde{\ell}_{L} \tilde{\nu}\right)= & 2 \operatorname{Re}\left\{\Delta_{i}\left(\tilde{\ell}_{L}\right) \Delta_{i}^{*}(\tilde{\nu}) g_{3}\right\}, \\
D_{i}(\tilde{\nu} \tilde{\nu})= & \left|\Delta_{i}(\tilde{\nu})\right|^{2} 2 g_{1} .
\end{aligned}
$$

The corresponding scalar products for the decay of the $\tilde{\chi}_{j}^{-}\left(p_{4}\right)$ and the expressions for $D_{j}(\alpha \beta)$, eq.(91), are obtained by the following substitutions:

$$
\begin{aligned}
& p_{5} \rightarrow p_{8}, p_{6} \rightarrow p_{9}, p_{7} \rightarrow p_{10}, \quad m_{i} \rightarrow m_{j}, m_{k} \rightarrow m_{l} \\
& O_{k i}^{L} \rightarrow O_{l j}^{L *}, O_{k i}^{R} \rightarrow O_{l j}^{R *} \\
& \Delta_{i}\left(\tilde{\ell}_{L}\right) \rightarrow \Delta_{j}\left(\tilde{\ell}_{L}\right), \quad \Delta_{i}(\tilde{\nu}) \rightarrow \Delta_{j}(\tilde{\nu}) \quad \text { (see eq.(23)). }
\end{aligned}
$$

\subsection{2 $\quad \Sigma_{a}^{D}(\alpha \beta)$ and $\Sigma_{b}^{D}(\alpha \beta)$}

These are the parts of eq.(87) and eq.(88) which contain the polarization vector either $s^{a}$ of $\tilde{\chi}_{i}^{+}$or $s^{b}$ of $\tilde{\chi}_{j}^{-}$. In the term $\Sigma_{a}^{D}(\alpha \beta)$ of eq.(90) the following five products with the polarization vector $s^{a}$ appear:

$g_{1}^{a}=m_{i}\left(p_{5} p_{7}\right)\left(p_{6} s^{a}\right)$,

$g_{2}^{a}=m_{i}\left(p_{5} p_{6}\right)\left(p_{7} s^{a}\right)$,

$g_{3}^{a}=m_{k}\left(p_{3} p_{7}\right)\left(p_{6} s^{a}\right)$,

$g_{4}^{a}=m_{k}\left(p_{3} p_{6}\right)\left(p_{7} s^{a}\right)$,

$g_{5}^{a}=m_{k}\left[s^{a} p_{3} p_{7} p_{6}\right]$. 


$$
\begin{aligned}
\Sigma_{a}^{D}\left(W^{+} W^{+}\right)= & |\Delta(W)|^{2} 4\left[\left(\left|O^{R}\right|^{2}-\left|O^{L}\right|^{2}\right)\left(g_{1}^{a}+g_{2}^{a}\right)-\left(O^{L *} O^{R}+O^{L} O^{R *}\right)\left(-g_{3}^{a}+g_{4}^{a}\right)\right. \\
& \left.-\left(\left|O^{R}\right|^{2}+\left|O^{L}\right|^{2}\right)\left(g_{1}^{a}-g_{2}^{a}\right)+\left(O^{L *} O^{R}-O^{L} O^{R *}\right) g_{5}^{a}\right] \\
\Sigma_{a}^{D}\left(W^{+} \tilde{\ell}_{L}\right)= & 2 \operatorname{Re}\left\{\Delta(W) \Delta_{i}^{*}\left(\tilde{\ell}_{L}\right) 2\left[2 O^{R} g_{2}^{a}-O^{L}\left(-g_{3}^{a}+g_{4}^{a}+g_{5}^{a}\right)\right]\right\} \\
\Sigma_{a}^{D}\left(W^{+} \tilde{\nu}\right)= & 2 \operatorname{Re}\left\{-\Delta(W) \Delta_{i}^{*}(\tilde{\nu}) 2\left[-2 O^{L} g_{1}^{a}-O^{R}\left(-g_{3}^{a}+g_{4}^{a}-g_{5}^{a}\right)\right]\right\} \\
\Sigma_{a}^{D}\left(\tilde{\ell}_{L} \tilde{\ell}_{L}\right)= & \left|\Delta_{i}\left(\tilde{\ell}_{L}\right)\right|^{2} 2 g_{2}^{a} \\
\Sigma_{a}^{D}\left(\tilde{\ell}_{L} \tilde{\nu}\right)= & 2 \operatorname{Re}\left\{-\Delta_{i}\left(\tilde{\ell}_{L}\right) \Delta_{i}^{*}(\tilde{\nu})\left(g_{3}^{a}-g_{4}^{a}+g_{5}^{a}\right)\right\} \\
\Sigma_{a}^{D}(\tilde{\nu} \tilde{\nu})= & \left|\Delta_{i}(\tilde{\nu})\right|^{2} 2\left(-g_{1}^{a}\right)
\end{aligned}
$$

The corresponding expressions $\Sigma_{b}^{D}(\alpha \beta)$, eq. (92), for the decay of $\tilde{\chi}_{j}^{-}\left(p_{4}\right)$ one obtains by the same substitutions as in 2.3.1, eqs. (102)-(104), and the additional substitution $s^{a} \rightarrow-s^{b}$ in eqs.(105)-(108) and $\left[s^{a} p_{3} p_{7} p_{6}\right] \rightarrow\left[\left(-s^{b}\right) p_{4} p_{10} p_{9}\right]^{*}$ in eq.(109).

\subsection{Amplitude squared for production and decay}

The amplitude squared $|T|^{2}$ of the combined processes of production and decays, eqs.(1) - (3), is given by:

$$
\begin{array}{r}
|T|^{2}=\sum_{(\alpha \beta)\left(\alpha_{i} \beta_{i}\right)\left(\alpha_{j} \beta_{j}\right)} 4\left(P(\alpha \beta) D_{i}\left(\alpha_{i} \beta_{i}\right) D_{j}\left(\alpha_{j} \beta_{j}\right)+\Sigma_{a}^{P}(\alpha \beta) \Sigma_{a}^{D}\left(\alpha_{i} \beta_{i}\right) D_{j}\left(\alpha_{j} \beta_{j}\right)\right. \\
\left.+\Sigma_{b}^{P}(\alpha \beta) \Sigma_{b}^{D}\left(\alpha_{j} \beta_{j}\right) D_{i}\left(\alpha_{i} \beta_{i}\right)+\Sigma_{a b}^{P}(\alpha \beta) \Sigma_{a}^{D}\left(\alpha_{i} \beta_{i}\right) \Sigma_{b}^{D}\left(\alpha_{j} \beta_{j}\right)\right) .
\end{array}
$$

The arguments label the six combinations of the exchanged particles:

$$
\begin{aligned}
& (\alpha \beta):(\gamma \gamma),(\gamma Z),(\gamma \tilde{\nu}),(Z Z),(Z \tilde{\nu}),(\tilde{\nu} \tilde{\nu}), \\
& \left(\alpha_{i} \beta_{i}\right):\left(W^{+} W^{+}\right),\left(W^{+} \tilde{\ell}_{L}\right),\left(W^{+} \tilde{\nu}\right),\left(\tilde{\ell}_{L} \tilde{\ell}_{L}\right),\left(\tilde{\ell}_{L} \tilde{\nu}\right),(\tilde{\nu} \tilde{\nu}), \\
& \left(\alpha_{j} \beta_{j}\right):\left(W^{-} W^{-}\right),\left(W^{-} \tilde{\ell}_{L}\right),\left(W^{-} \tilde{\nu}\right),\left(\tilde{\ell}_{L} \tilde{\ell}_{L}\right),\left(\tilde{\ell}_{L} \tilde{\nu}\right),(\tilde{\nu} \tilde{\nu}) .
\end{aligned}
$$

The first product in eq.(116) is the part obtained by neglecting all spin correlations between production and decay. The second and third term describe the correlations between the production and the decay process either of $\tilde{\chi}_{i}^{+} \rightarrow \tilde{\chi}_{k}^{0} \ell^{+} \nu_{\ell}$ or $\tilde{\chi}_{j}^{-} \rightarrow \tilde{\chi}_{\ell}^{0} \ell^{-} \bar{\nu}_{\ell}$, and in the last term correlations between both decay processes are included.

- In the first term of eq.(116) only scalar products appear which can be expressed by the Mandelstam variables $s, t, u$ for the production and decay processes.

- To obtain the second (third) term of eq.(116) one has to calculate all combinations of $f_{m}^{a} \times g_{n}^{a}, m, n=1, \ldots, 5\left(f_{m}^{b} \times g_{n}^{b}, m, n=1, \ldots, 5\right)$ using eq.(31). In this way one gets additional scalar products:

$\left(p_{1,2} p_{6,7}\right), \quad\left(p_{1,2} p_{9,10}\right)$,

describing correlations between production and decay. These scalar products can not be expressed by Mandelstam variables. They contain the angle between the 
incoming electron and the outgoing lepton in the laboratory system. The combinations $\left[s^{(a, b)} p_{k} p_{l} p_{m}\right]$, eqs.(60), (71), (109), are due to complex parameters and to the term in the propagators, eq.(23), containing the width of the exchanged particle. They lead to triple product correlations (using eq.(31)):

$\left[p_{1,2} p_{3} p_{7} p_{6}\right], \quad\left[p_{1,2} p_{4} p_{10} p_{9}\right], \quad\left[p_{6,7} p_{2} p_{1} p_{3}\right], \quad\left[p_{9,10} p_{2} p_{1} p_{4}\right]$

- To obtain the last term of eq.(116) one has to calculate all combinations of $f_{m}^{a b} \times$ $g_{n_{i}}^{a} \times g_{n_{j}}^{b}, m=1, \ldots, 8, n_{i}, n_{j}=1, \ldots, 5$ using again eq.(31). Then due to the combinations $\left(s^{a} s^{b}\right)$, eq. $\left([77)\right.$, and $\left[s^{a} s^{b} p_{k} p_{l}\right]$, eq.(80), also correlations between the decay products of both charginos appear:

$\left(p_{6,7} p_{9,10}\right), \quad\left[p_{6,7} p_{4} p_{10} p_{9}\right], \quad\left[p_{9,10} p_{3} p_{7} p_{6}\right], \quad\left[p_{9,10} p_{6,7} p_{3} p_{1}\right], \quad\left[p_{9,10} p_{6,7} p_{2} p_{4}\right]$.

If only the decay of one chargino, e.g. $\tilde{\chi}_{j}^{-}$is considered, one has to sum over the spin of $\tilde{\chi}_{i}^{+}$so that in eq. (116) $D_{i}\left(\alpha_{i} \beta_{i}\right)=1$ and $\Sigma_{a}^{D}\left(\alpha_{i} \beta_{i}\right)=0$.

\section{Numerical Results and Discussion}

In the MSSM [9] the masses and couplings of charginos and neutralinos are determined by the parameters $M^{\prime}, M, \mu, \tan \beta$, with $M^{\prime}$ usually fixed by the GUT relation $M^{\prime}=$ $\frac{5}{3} M \tan ^{2} \Theta_{W}$. Since we do not consider CP violation in the following analysis, the parameters and the couplings of charginos and neutralinos can be chosen real. The neutralino and chargino mass mixing matrices can be found in [9, 13].

The masses of the sleptons and of the sneutrinos are related to the parameters $M$ and $\tan \beta$ of the MSSM and to the common scalar mass $m_{0}$ at the unification point by the renormalization group equations [14]:

$m_{\tilde{f}_{L}}^{2}=m_{0}^{2}+0.79 M^{2}+m_{Z}^{2} \cos 2 \beta\left(T_{3}^{f}-Q_{f} \sin ^{2} \Theta_{W}\right)$.

Here $T_{3}^{f}$ and $Q_{f}$ denote the third component of the weak isospin and the charge of the corresponding fermion.

In the following numerical analysis we study the pair production of the lighter chargino $\tilde{\chi}_{1}^{ \pm}$with unpolarized beams, where one of the charginos decays leptonically, $\tilde{\chi}_{1}^{-} \rightarrow \tilde{\chi}_{1}^{0}+$ $\ell^{-}+\bar{\nu}_{\ell}$. In order to illustrate the influence of the gaugino-higgsino mixing of $\tilde{\chi}_{1}^{ \pm}$and $\tilde{\chi}_{1}^{0}$ we consider four representative scenarios.

In Table 1 we give the parameters and the mass eigenvalues (including their sign). For the scalar mass parameter we choose in general $m_{0}=200 \mathrm{GeV}$. Since the angular distribution depends also on the value of $m_{0}$, we compare in scenario $(\mathrm{B})$ the numerical results for $m_{0}=200 \mathrm{GeV}$ with those for $m_{0}=100 \mathrm{GeV}$. In Table 2 the gaugino and higgsino components of the chargino and of $\tilde{\chi}_{1}^{0}$ are given.

In scenarios $(\mathrm{A})$ and $(\mathrm{B})$ for low and high $\tan \beta$, respectively, $\tilde{\chi}_{1}^{ \pm}$is almost a pure $\mathrm{W}$-ino and $\tilde{\chi}_{1}^{0}$ is almost a pure B-ino. In scenarios (C) and (D) for low and high $\tan \beta$, respectively, both $\tilde{\chi}_{1}^{ \pm}$and $\tilde{\chi}_{1}^{0}$ have dominating higgsino components. 
In the following we will discuss the angular distribution $d \sigma / d \cos \Theta_{-}$(where $\Theta_{-}$is the angle between the outgoing $\ell^{-}$and the electron beam) and the forward-backward asymmetry

$$
A_{F B}=\frac{\sigma\left(\cos \Theta_{-}>0\right)-\sigma\left(\cos \Theta_{-}<0\right)}{\sigma\left(\cos \Theta_{-}>0\right)+\sigma\left(\cos \Theta_{-}<0\right)}
$$

of the lepton $\ell^{-}$from the leptonic decay $\tilde{\chi}_{1}^{-} \rightarrow \tilde{\chi}_{1}^{0}+e^{-}+\bar{\nu}$ for $\sqrt{s}=192 \mathrm{GeV}$ and $\sqrt{s}=200 \mathrm{GeV}$.

The total cross sections are given in Table 3 for $\sqrt{s}=192 \mathrm{GeV}$ and in Table 4 for $\sqrt{s}=200 \mathrm{GeV}$. They are independent of the spin correlations and factorize into the chargino production cross section times the leptonic branching ratio of $\tilde{\chi}_{1}^{-}$[5]. For comparison the different angular distributions in Figs. 4, 5 and 6 are normalized to the total cross section.

To demonstrate the significance of spin correlations we compare in Figs. 2 and 3 the angular distributions for $\tan \beta=40$ and $\sqrt{s}=200 \mathrm{GeV}$ with the results one obtains by factorizing the process in production and decay. The spin effect is sizeable for the gaugino-like charginos of scenario (B), Fig. 2. It is largest in the forward and in the backward direction. The forward-backward asymmetry is $A_{F B}=+39.5 \%$, one order of magnitude larger as that obtained neglecting the spin correlations between production and decay. For the higgsino-like scenario (D), Fig. 3, the influence of spin correlations is less significant. Quite generally, the influence of spin correlations is much more pronounced for gaugino-like charginos than for higgsino-like ones.

Especially for gaugino-like charginos the spin effects depend sensitively on the value of $\tan \beta$. For $\tan \beta=3$ they are smaller than for $\tan \beta=40$. For scenario (A) with $\tan \beta=3$ the forward-backward asymmetry is $A_{F B}=+6.3 \%\left(A_{F B}=7.9 \%\right)$ with spin correlations and $A_{F B}=2.0 \%\left(A_{F B}=3.8 \%\right)$ without spin correlations at $\sqrt{s}=192 \mathrm{GeV}$ $(\sqrt{s}=200 \mathrm{GeV})$. In Fig. 4 we compare the angular distributions for gaugino-like charginos for $\sqrt{s}=192 \mathrm{GeV}$ and $m_{0}=200 \mathrm{GeV}$ and two values of $\tan \beta, \tan \beta=3$, scenario (A), and $\tan \beta=40$, scenario (B). For both values of $\tan \beta$ the forward hemisphere is favoured, and the forward-backward asymmetry $A_{F B}$ is $+6.3 \%$ for $\tan \beta=3$ and $+38.9 \%$ for $\tan \beta=40$.

In contrast, for the higgsino-like charginos in scenario (C) and (D), Fig. 5, the distributions are rather flat with the backward direction somewhat favoured. The forwardbackward asymmetry is very small: $A_{F B}=-1.6 \%$ for $\tan \beta=3$, and $A_{F B}=-3.4 \%$ for $\tan \beta=40$. The shape of the angular distribution is similar at $\sqrt{s}=200 \mathrm{GeV}$ with the same values of $A_{F B}$ as for $\sqrt{s}=192 \mathrm{GeV}$ (see Table 4 ).

In the case of gaugino-like charginos the size of the forward-backward asymmetry depends on the value of $m_{0}$. In Fig. 6 we therefore compare for scenario (B) the angular distributions for $m_{0}=100 \mathrm{GeV}$ and $m_{0}=200 \mathrm{GeV}$. For $m_{0}=200 \mathrm{GeV}$ the forwardbackward asymmetry is $A_{F B}=+39.5 \%$ and for $m_{0}=100 \mathrm{GeV}$ the asymmetry is $A_{F B}=$ $+29.1 \%$. 


\section{Summary and conclusions}

We have calculated the analytical expression for the differential cross section for $e^{+} e^{-} \rightarrow$ $\tilde{\chi}_{i}^{+} \tilde{\chi}_{j}^{-}$with polarized beams and the subsequent leptonic decays $\tilde{\chi}_{i}^{+} \rightarrow \tilde{\chi}_{k}^{0} \ell^{+} \nu_{\ell}$ and $\tilde{\chi}_{j}^{-} \rightarrow$ $\tilde{\chi}_{l}^{0} \ell^{-} \bar{\nu}_{\ell}$, taking into account the complete spin correlations between production and decays. The differential cross section is composed of the joint spin-density matrix of the two charginos and the decay matrices for their leptonic decays. The corresponding expressions also include spin correlations between the leptons coming from the decays of both charginos. Moreover, the analytical formulae for the production and the decay matrices can be used as building blocks for processes with other chargino decay channels or for processes with other chargino production mechanisms, as e.g. $e^{-} \gamma$ or $\gamma \gamma$ collisions.

In the numerical analysis we have studied the pair production of the lighter chargino $\tilde{\chi}_{1}^{ \pm}$with unpolarized beams and to the leptonic decay of one of them, $\tilde{\chi}_{1}^{-} \rightarrow \tilde{\chi}_{1}^{0}+\ell^{-}+\bar{\nu}$. We have calculated the angular distribution of $\ell^{-}$in the laboratory frame at $\sqrt{s}=192 \mathrm{GeV}$ and $\sqrt{s}=200 \mathrm{GeV}$ for four representative MSSM scenarios which differ in the mixing character of the chargino and in the parameter $\tan \beta$. For the case of a gaugino-like $\tilde{\chi}_{1}^{-}$ we have also studied the influence of the scalar mass parameter $m_{0}$ on the shape of the angular distribution. Generally, the effect of spin correlations is much more significant for gaugino-like charginos than for higgsino-like ones.

The shape of the lepton angular distribution and the size of the forward-backward asymmetry depend on the nature of the charginos. For higgsino-like charginos the angular distribution is nearly flat with a small forward-backward asymmetry, whereas for gauginolike ones the forward-backward asymmetry is between $6.3 \%$ and $39.5 \%$ for $m_{0}=200 \mathrm{GeV}$. In the case of gaugino-like charginos, however, the shape of the angular distribution and the forward-backward asymmetry depend on the scalar mass $m_{0}$.

In conclusion, we have found that for a precise analysis of the lepton angular distributions in the decays of charginos produced in $e^{+} e^{-} \rightarrow \tilde{\chi}_{i}^{+} \tilde{\chi}_{j}^{-}$the inclusion of spin correlations between production and decay is necessary for gaugino-like charginos.

\section{Acknowledgement}

We thank H. Wachter for checking the analytical formulae and we are grateful to V. Latussek for his support in the development of the numerical program. G.M.-P. was supported by Friedrich-Ebert-Stiftung. This work was also supported by the German Federal Ministry for Research and Technology (BMBF) under contract number 05 7WZ91P (0), by the Deutsche Forschungsgemeinschaft under contract Fr 1064/2-2 and the 'Fond zur Förderung der wissenschaftlichen Forschung' of Austria, Project No. P10843-PHY.

\section{References}

[1] A. Bartl, H. Fraas, W. Majerotto, B. Mößlacher, Z.Phys. C 55 (1992) 257.

A. Bartl, H. Fraas, W. Majerotto, Z.Phys. C 30 (1986) 441. 
[2] M. Chen, C. Dionisi, M. Martinez, X. Tata, Phys.Rep. 159 (1988) 201.

S. Ambrosanio et al., in Physics at LEP2, CERN 96-01, Vol. 1, p. 463, eds. G. Altarelli, T. Sjöstrand and F. Zwirner.

[3] A. Bartl, W. Majerotto, B. Mößlacher, in ' $e^{+} e^{-}$Collisions at $500 \mathrm{GeV}$ : The Physics Potential', Part B, DESY 92-123B, p. 641, ed. by P.M. Zerwas.

[4] S. Kawasaki, T. Shirafuji, S.Y. Tsai, Progress of Theor. Phys., 49 (1973) 1656.

[5] D.A. Dicus, E.C.G. Sudarshan, X. Tata, Phys. Lett. B 154 (1985) 79.

[6] J.L. Feng, M.J. Strassler, Phys. Rev. D 55 (1997) 1326.

[7] S.Y. Choi, hep-ph/9801323.

[8] DFGT: C. Dionisi, K. Fujii, S. Giagu, T. Tsukamoto, in Physics at LEP2, CERN 96-01, Vol. 2, p. 337, eds. G. Altarelli, T. Sjöstrand and F. Zwirner.

SUSYGEN: S. Katsanevas, S. Melachroinos, in Physics at LEP2, CERN 96-01, Vol. 2, p. 328, eds. G. Altarelli, T. Sjöstrand and F. Zwirner.

CompHEP: A.S. Belyaev, A.V. Gladyshev, A.V. Semenov, hep-ph/9712303.

GRACE: M. Jimbo et al., hep-ph/9503363, hep-ph/9503365.

[9] H.E. Haber, G.L. Kane, Phys. Rep. 117 (1985) 75.

[10] G. Moortgat-Pick, H. Fraas, hep-ph/9708481, submitted to Phys.Rev.D.

[11] G. Moortgat-Pick, H. Fraas, A. Bartl, W. Majerotto, hep-ph/9803304, to be published in Act. Phys. Pol. B.

[12] H.E. Haber, Proceedings of the 21st SLAC Summer Institute on Particle Physics, Stanford 1993, p. 231, eds. L. DePorcel, Ch. Dunwoodie.

[13] A. Bartl, H. Fraas, W. Majerotto, Phys. Rev. D 40 (1989) 1594.

[14] L.J. Hall, J. Polchinski, Phys. Lett. B 152 (1985) 335.

[15] G. Moortgat-Pick, H. Fraas, Act. Phys. Pol. B 28 (1997) 2395. 


\begin{tabular}{|c||c|c|c|c|c|c|c|c|} 
& $\mathrm{M}$ & $\mu$ & $\tan \beta$ & $m_{0}$ & $m_{\tilde{\chi}_{1}^{0}}$ & $m_{\tilde{\chi}_{1}^{ \pm}}$ & $m_{\tilde{e}_{L}}$ & $m_{\tilde{\nu}}$ \\
\hline \hline $\mathrm{A}$ & 87 & -800 & 3 & 200 & 45 & 91 & 219 & 207 \\
\hline $\mathrm{B}$ & 91.5 & -800 & 40 & $200(100)$ & 45 & 91 & $221(137)$ & $206(112)$ \\
\hline $\mathrm{C}$ & 363 & 107 & 3 & 200 & 76 & -91 & 382 & 375 \\
\hline $\mathrm{D}$ & 302 & 99 & 40 & 200 & 75 & -91 & 338 & 328 \\
\hline
\end{tabular}

Table 1: Parameters $\mathrm{M}, \mu, \tan \beta$, and $m_{0}$, and the resulting mass eigenvalues. All masses are given in $[\mathrm{GeV}]$.

\begin{tabular}{|c|cc|cc|cccc|} 
& \multicolumn{2}{|c|}{$\tilde{\chi}_{1}^{+}$} & \multicolumn{2}{|c|}{$\tilde{\chi}_{1}^{-}$} & \multicolumn{4}{|c|}{$\tilde{\chi}_{1}^{0}$} \\
\hline & $\left(w^{+} \mid\right.$ & $\left.H^{+}\right)$ & $\left(w^{-} \mid\right.$ & $\left.H^{-}\right)$ & $(\tilde{\gamma} \mid$ & $\tilde{Z} \mid$ & $\tilde{H}_{a}^{0} \mid$ & $\left.\tilde{H}_{b}^{0}\right)$ \\
\hline $\mathrm{A}$ & $(+1.0 \mid$ & $+.03)$ & $(+.99 \mid$ & $+.13)$ & $(+.90 \mid$ & $-.44 \mid$ & $-.03 \mid$ & $-.04)$ \\
\hline $\mathrm{B}$ & $(+1.0 \mid$ & $-.01)$ & $(+.99 \mid$ & $+.14)$ & $(+.87 \mid$ & $-.48 \mid$ & $+.0004 \mid$ & $-.05)$ \\
\hline $\mathrm{C}$ & $(+.33 \mid$ & $-.95)$ & $(-.18 \mid$ & $+.98)$ & $(+.18 \mid$ & $-.35 \mid$ & $+.78 \mid$ & $+.48)$ \\
\hline $\mathrm{D}$ & $(+.38 \mid$ & $-.92)$ & $(-.13 \mid$ & $+.99)$ & $(-.19 \mid$ & $+.34 \mid$ & $-.58 \mid$ & $-.72)$ \\
\hline
\end{tabular}

Table 2: Mixing character of the chargino $\tilde{\chi}_{1}^{ \pm}$and the neutralino $\tilde{\chi}_{1}^{0}$.

\begin{tabular}{|l||c|c|c|c|} 
& $\mathrm{A}$ & $\mathrm{B}$ & $\mathrm{C}$ & $\mathrm{D}$ \\
\hline \hline$\sigma_{t} / \mathrm{fb}$ & 339 & 587 & 177 & 175 \\
\hline$A_{F B}$ & +.063 & +.389 & -.016 & -.034 \\
\hline
\end{tabular}

Table 3: $\sigma\left(e^{-} e^{+} \rightarrow \tilde{\chi}_{1}^{+} \tilde{\chi}_{1}^{-}\right) \times \mathrm{BR}\left(\tilde{\chi}_{1}^{-} \rightarrow \tilde{\chi}_{1}^{0} \ell^{-} \bar{\nu}\right)$ and forward-backward asymmetries $A_{F B}$, eq.(124), for $\sqrt{s}=192 \mathrm{GeV}$.

\begin{tabular}{|l||c|c|c|c|c|} 
& $\mathrm{A}$ & $\mathrm{B}, m_{0}=200 \mathrm{GeV}$ & $\mathrm{B}, m_{0}=100 \mathrm{GeV}$ & $\mathrm{C}$ & $\mathrm{D}$ \\
\hline \hline$\sigma_{t} / \mathrm{fb}$ & 368 & 638 & 177 & 204 & 202 \\
\hline$A_{F B}$ & +.079 & +.395 & .291 & -.016 & -.034 \\
\hline
\end{tabular}

Table 4: $\sigma\left(e^{-} e^{+} \rightarrow \tilde{\chi}_{1}^{+} \tilde{\chi}_{1}^{-}\right) \times \operatorname{BR}\left(\tilde{\chi}_{1}^{-} \rightarrow \tilde{\chi}_{1}^{0} \ell^{-} \bar{\nu}\right)$ and forward-backward asymmetries $A_{F B}$, eq.(124), for $\sqrt{s}=200 \mathrm{GeV}$. 

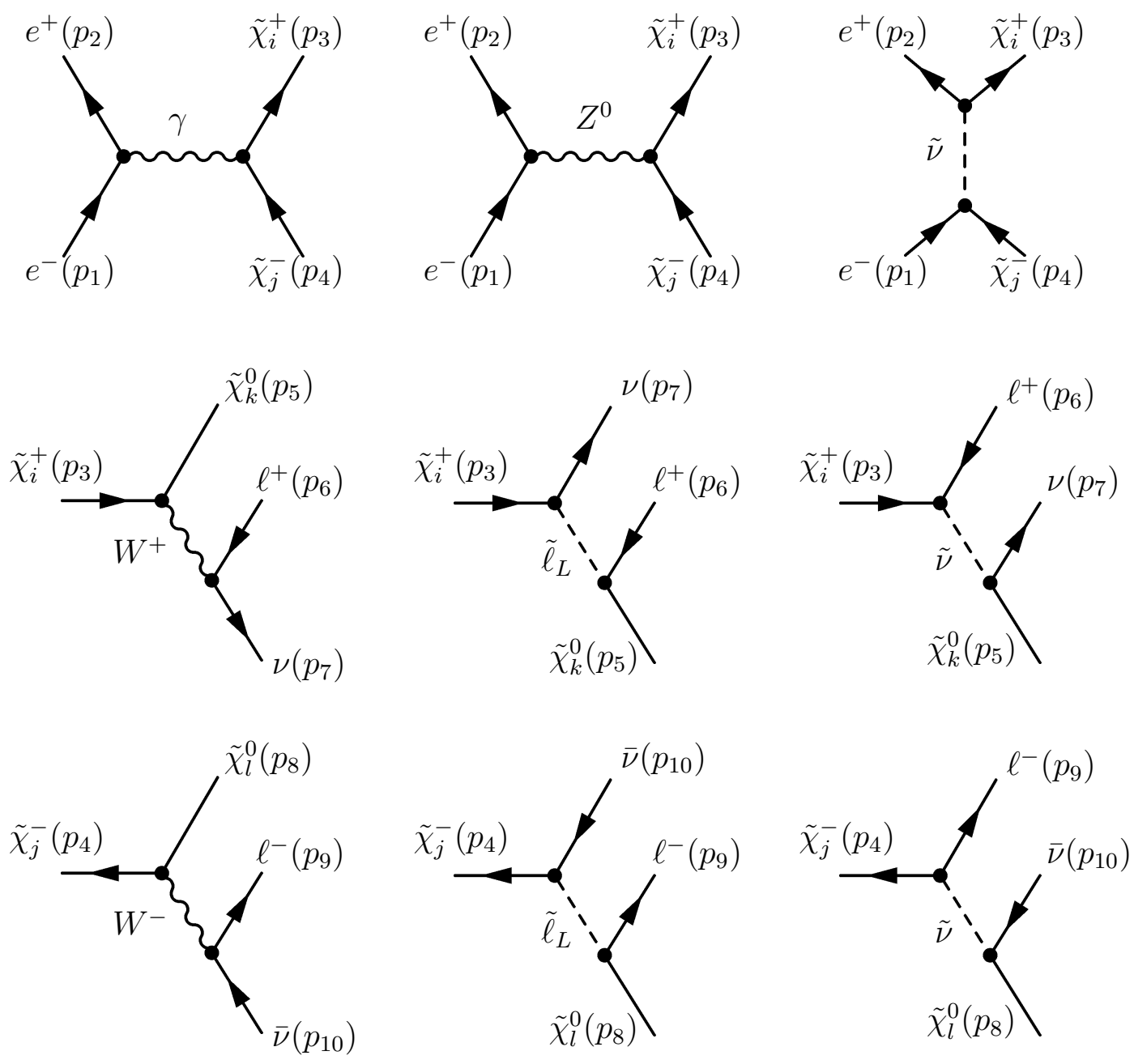

Figure 1: Feynman graphs for production and leptonic decays of charginos. 


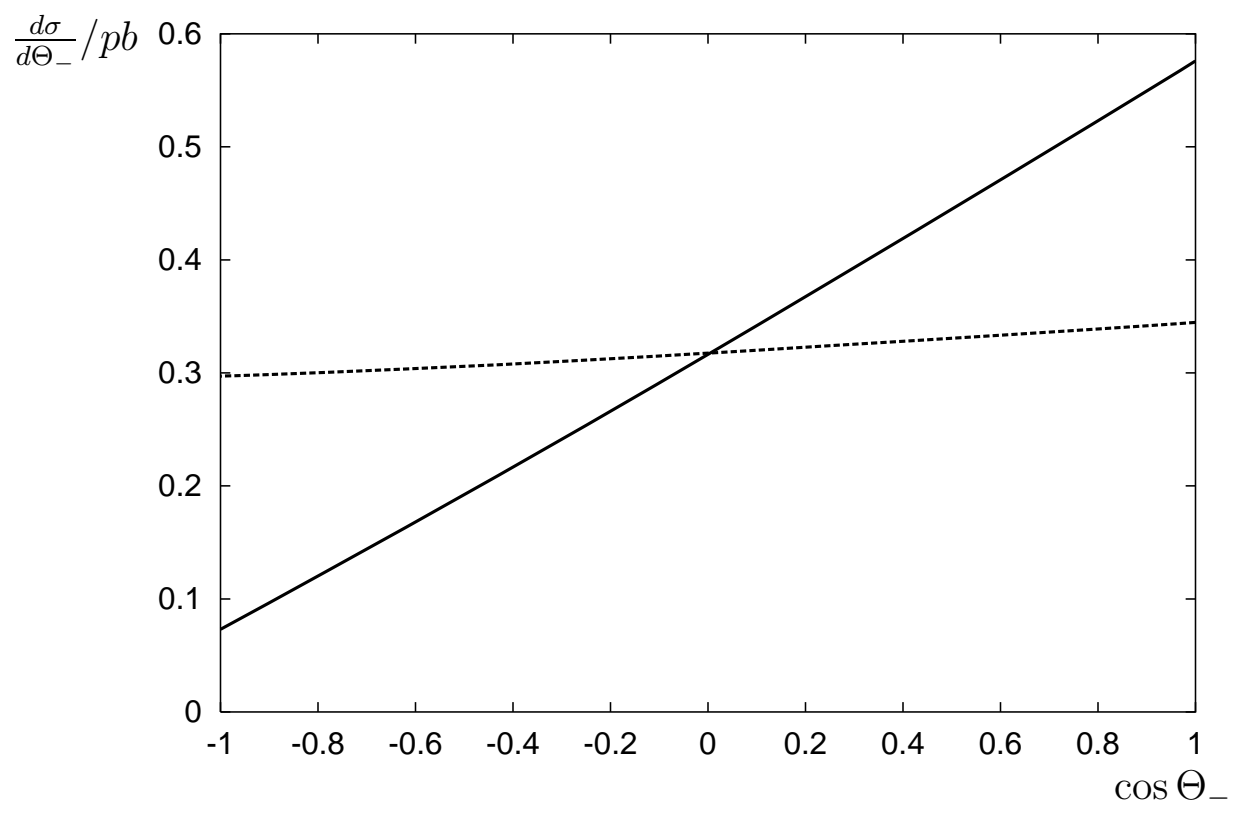

Figure 2: Lepton angular distributions in scenario (B) for $m_{0}=200 \mathrm{GeV}$ at $\sqrt{s}=200$ $\mathrm{GeV}$ with spin correlations (solid) and without spin correlations (dotted).

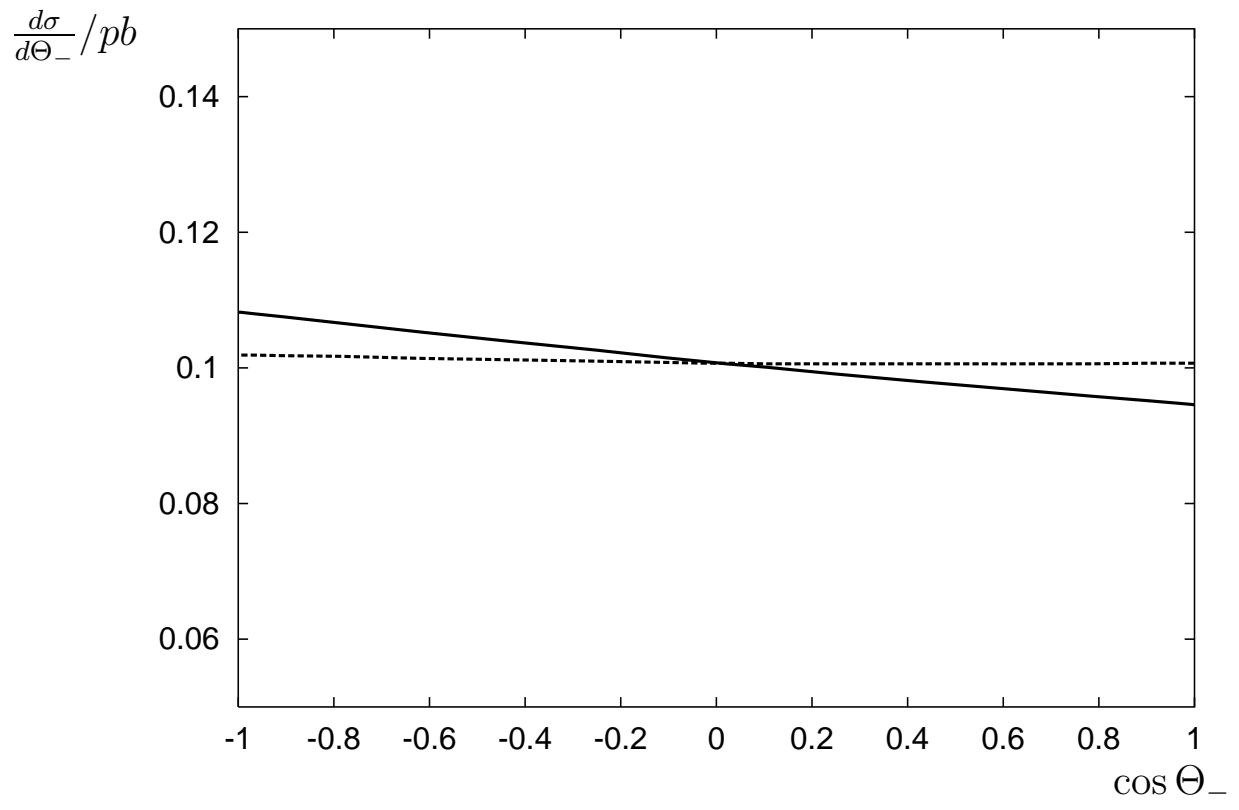

Figure 3: Lepton angular distribution in scenario (D) at $\sqrt{s}=200 \mathrm{GeV}$ with spin correlations (solid), and without spin correlations (dotted). 


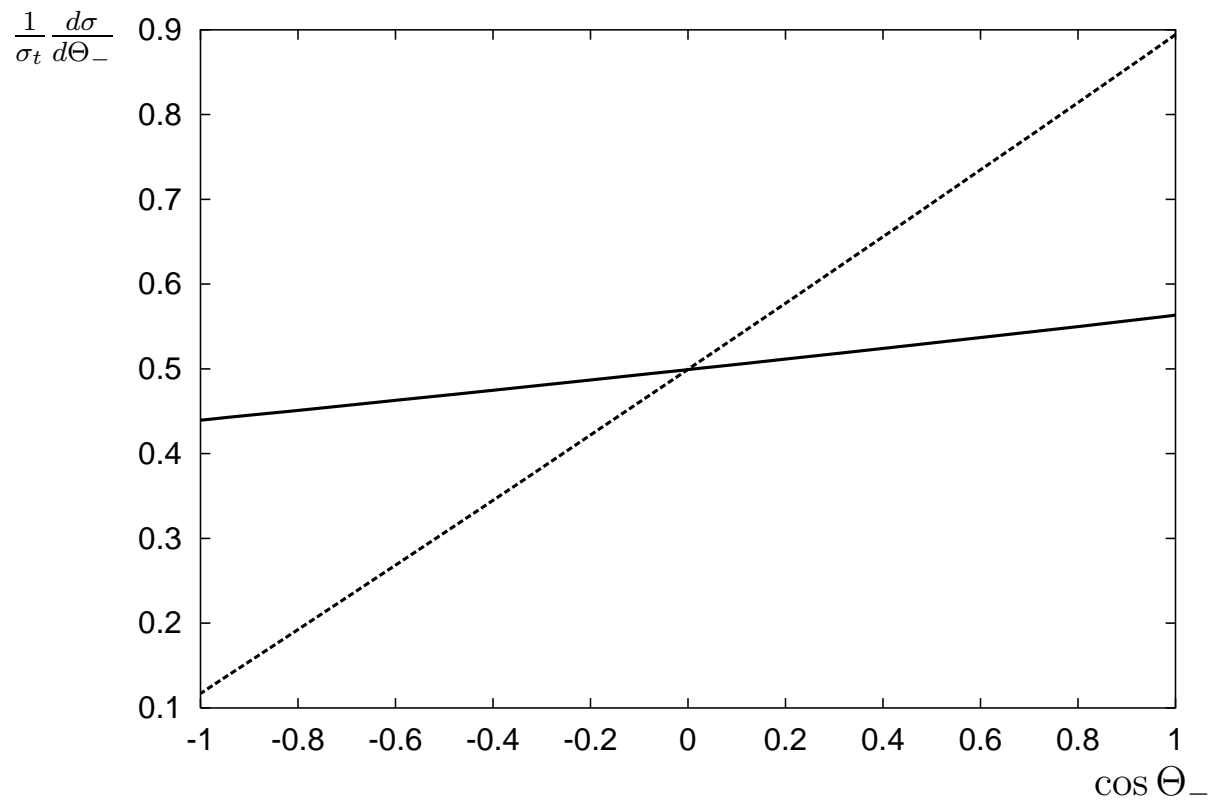

Figure 4: Lepton angular distributions in scenario (A) (solid) and scenario (B) (dotted) for $\sqrt{s}=192 \mathrm{GeV}$, normalized to the total cross section.

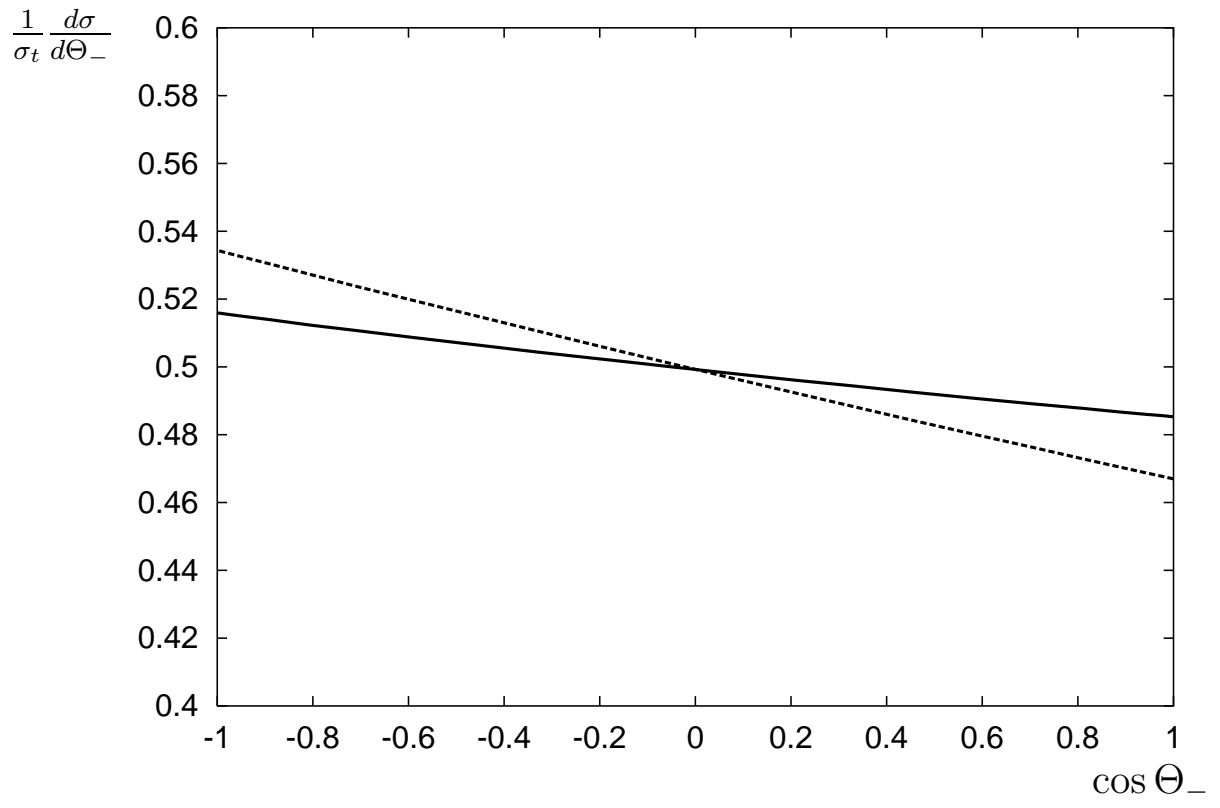

Figure 5: Lepton angular distribution in scenario (C) (solid) and scenario (D) (dotted) at $\sqrt{s}=192 \mathrm{GeV}$, normalized to the total cross section. 


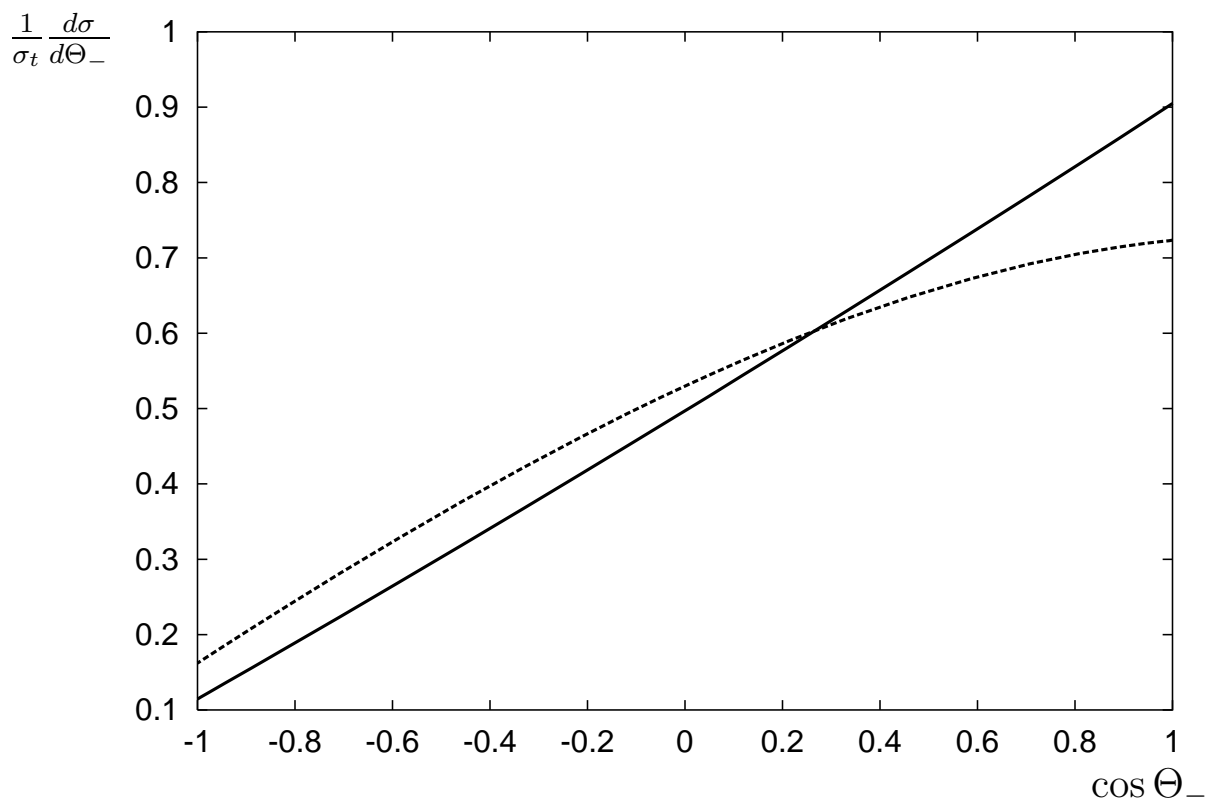

Figure 6: Lepton angular distribution in scenario (B) at $\sqrt{s}=200 \mathrm{GeV}$ with $m_{0}=$ $200 \mathrm{GeV}$ (solid) and $m_{0}=100 \mathrm{GeV}$ (dotted), normalized to the total cross section. 